\title{
Assessing transpulmonary pressure via direct pleural manometry
}

\author{
Christopher Rugg ${ }^{*}$ (D), Stefan Schmid, Janett Kreutziger and Mathias Ströhle
}

To the Editor,

With great interest we read the multicentre, prospective study on PEEP titration conducted in ARDS patients by Bergez et al. [1]. PEEP was set on the basis of two cleverly devised protocols-one referring to plateau the other to transpulmonary pressure $\left(P_{\mathrm{L}}\right)$. Both were able to improve oxygenation compared to baseline settings with the latter slightly increasing possible over-distension. In accordance with an also recently published expert review by Gattinoni et al., they recognized diverse limitations and assumptions while using esophageal pressure $\left(P_{\text {es }}\right)$ as a surrogate for pleural pressure $\left(P_{\mathrm{pl}}\right)$ [2]. Nonetheless, another animal and human cadaver study revealed that $P_{\text {es }}$ accurately reflects $P_{\mathrm{pl}}$ in the mid to dependent lung [3]. Regarding studies on mechanical ventilation, direct measurement of $P_{\mathrm{pl}}$ is usually restricted to animal or cadaver studies and is typically described as not feasible in clinical practice [2]. In 2018, a detailed review by Zielinska-Krawczyk et al. revealed that regarding therapeutic thoracenteses literature on direct pleural manometry actually is quite copious [4]. Direct pleural manometry has been used for studying various pathophysiological aspects of thoracentesis including the safety of high-volume pleural fluid removal. Furthermore, a portable electronic manometer, easy to build and only using widely accessible elements has been described [5]. Therefore, a small-bore catheter, inserted into the pleural cavity was connected with a vascular pressure transducer via

This comment refers to the article available at https://doi.org/10.1186/s1361 3-019-0554-3.

*Correspondence: christopher.rugg@tirol-kliniken.at

Department of General and Surgical Critical Care Medicine, Medical

University of Innsbruck, Anichstrasse 35, 6020 Innsbruck, Austria a 3-way stopcock. After referencing the pressure to the catheter insertion point into the chest, $P_{\mathrm{pl}}$ was precisely measured.

We would like to share our experience in performing such measurements in an ICU setting. From a total of over 600 patients per year in our department (23 beds, mixed ICU), over 100 receive pleural pigtail-catheters, mostly indicated for gas exchange-limiting pleural effusions. These patients are usually decompensated of heart or pulmonary failure, intubated and to some extent mechanically ventilated. Our anatomical landmark for catheter insertion is the inferior angle of the scapula, with the patient in lateral position. The catheter tip then usually lies dorsally in the middle to caudal section of the pleural cavity, with position controlled by chest radiography and sonography. Awareness of catheter tip position is fundamental for adequate interpretation of measured pressures, as is the fact that pleural surface pressure may differ from pleural fluid pressure [4]. Measurements can be intermittently displayed on our standard ICU monitor as a pressure curve for example, monitoring also negative values (Fig. 1). Noteworthy is that the units displayed are usually $\mathrm{mmHg}$ and one must convert to $\mathrm{cmH}_{2} \mathrm{O}$. In so doing we are able to conduct reliable, real-time measurements of $P_{\mathrm{pl}}$ during the complete respiratory cycle, regardless of ventilation mode. Complications are scarce, include pneumo- or haematothorax and occur mainly during insertion or removal of the catheter.

Of course, in critically ill patients pleural effusions are not always present, but if they are, drainage with a small-bore catheter not only helps improve oxygenation, but also gives a possibility to easily and directly measure $P_{\mathrm{pl}}$ and thus titrate ventilator settings without further deployment of additional devices. This can be beneficial in settings of limited resources. Direct pleural 


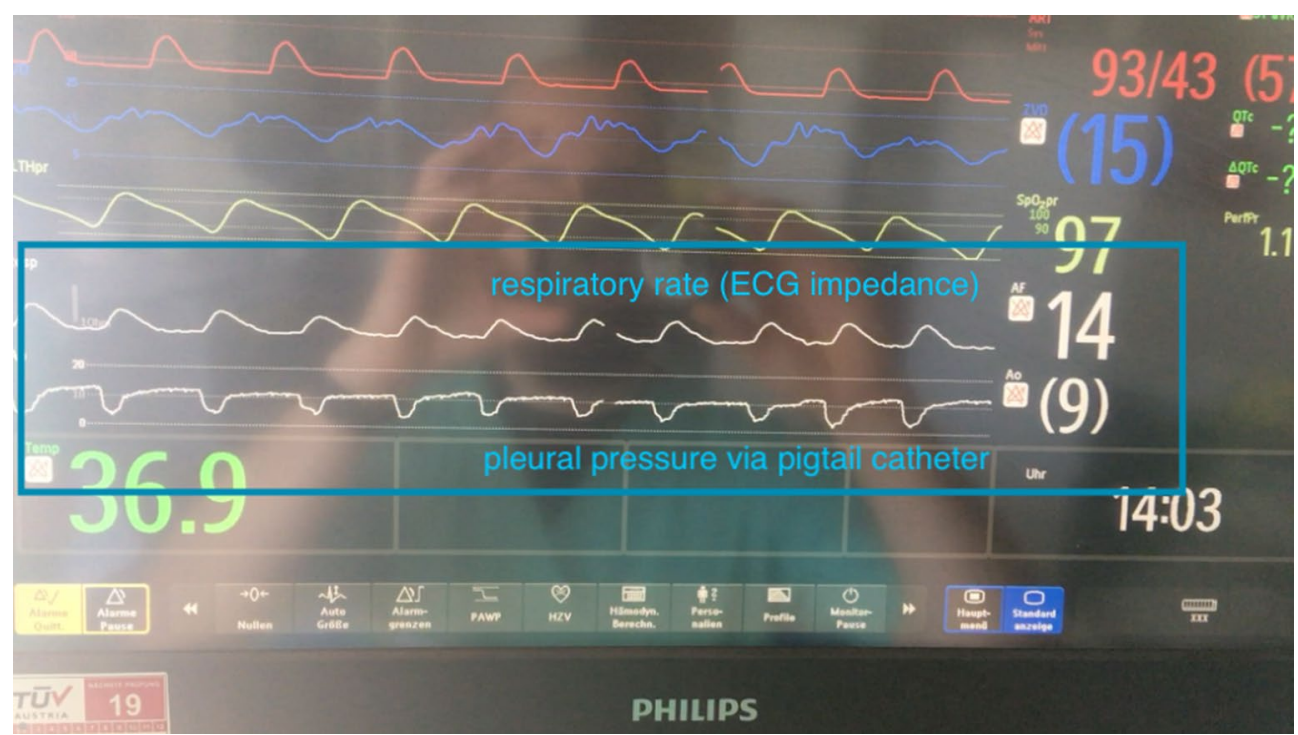

Fig. 1 Direct pleural manometry of an intubated, spontaneously breathing patient in an ICU setting

manometry via thoracic pigtail-catheters can be seen as an alternative to rather than a replacement of esophageal measurements in a special subgroup of patients.

\section{Abbreviations}

$P_{\mathrm{L}}:$ Transpulmonary pressure; $P_{\mathrm{es}}$ : Esophageal pressure; $P_{\mathrm{pl}}$ : Pleural pressure.

\section{Acknowledgements}

Not applicable.

\section{Authors' contributions}

CR, SS, JK and MS critically read the original paper by Bergez et al. CR and MS reviewed the literature on pleural manometry and adapted the technical set-up for the ICU setting. SS and JK verified the final set-up. First draft by CR. SS, JK and MS all contributed to the final draft. All authors read and approved the final manuscript.

\section{Funding}

None.

\section{Availability of data and materials}

Not applicable.

\section{Ethics approval and consent to participate}

Not applicable.

\section{Consent for publication}

Not applicable.

\section{Competing interests}

The authors declare that they have no competing interests.
Received: 8 October 2019 Accepted: 20 July 2020

Published online: 03 August 2020

\section{References}

1. Bergez M, Fritsch N, Tran-Van D, Saghi T, Bounkim T, Gentile A, Labadie P, Fontaine B, Ouattara A, Rozé H. PEEP titration in moderate to severe ARDS: plateau versus transpulmonary pressure. Ann Intensive Care. 2019;9:81.

2. Gattinoni L, Giosa L, Bonifazi M, Pasticci I, Busana M, Macri M, Romitti F, Vassalli F, Quintel M. Targeting transpulmonary pressure to prevent ventilator-induced lung injury. Expert Rev Respir Med. 2019;13(8):737-46.

3. Yoshida T, Amato MBP, Grieco D, Chen L, Lima CAS, Roldan R, Morais CCA, Gomes S, Costa ELV, Cardoso PFG, Charbonney E, Richard JCM, Brochard L, Kavanagh BP. Esophageal manometry and regional transpulmonary pressure in lung injury. Am J Respir Crit Care Med. 2018;197:1018-26.

4. Zielinska-Krawczyk M, Krenke R, Grabczak EM, Light RW. Pleural manometry-historical background, rationale for use and methods of measurement. Respir Med. 2018;136:21-8.

5. Krenke R, Guć M, Grabczak EM, Michnikowski M, Pałko KJ, Chazan R Gólczewski T. Development of an electronic manometer for intrapleural pressure monitoring. Respiration. 2011;136:21-8.

\section{Publisher's Note}

Springer Nature remains neutral with regard to jurisdictional claims in published maps and institutional affiliations. 